\title{
CLARIFYing the differences between men and women with stable CAD
}

A comparison of the risk profiles and 1 -year outcomes of 23,975 men and 7,002 women with stable coronary artery disease (CAD) was presented at the 2012 European Society of Cardiology Congress in late August and published in the European Heart Journal. Assessed patients were enrolled in CLARIFY - a multinational, prospective, observational, longitudinal registry of 33,285 outpatients with stable CAD.

At baseline, the women were older and were more likely to have diabetes mellitus, treated hypertension, symptoms of angina or heart failure (which were also more severe), and/or presence of myocardial ischemia on noninvasive testing than the CLARIFY men. Additionally, the women had less-severe CAD, were less likely to have a history of myocardial infarction, PCI, and/or CABG surgery, and were less likely to have undergone coronary angiography or noninvasive testing for myocardial ischemia than their male counterparts. Few differences in treatments were noted.
At 1-year follow-up, unadjusted event rates were similar for the men and women, except for a higher rate of unstable angina and a lower rate of revascularization in the female cohort. After adjustment for baseline characteristics, all assessed event rates were similar for the two cohorts, except for revascularization, which was again shown to have occurred less often in the women.

The investigators point out that "despite the very large size of [CLARIFY], event rates are somewhat low and ... one cannot rule out an actual increase in risk of cardiovascular event rates of $\sim 15 \%$ ". Nevertheless, they conclude that their findings demonstrate "similar crude and adjusted rates of mortality and cardiovascular events in men and women with stable CAD".

Bryony M. Mearns

Original article Steg, P. G. et al. Women and men with stable coronary artery disease have similar clinical outcomes: insights from the international prospective CLARIFY registry. Eur. Heart J. doi:10.1093/eurheartj/ehs289 